\title{
Origin of steps in magnetization loops of martensitic Ni-Mn-Ga films on $\mathrm{MgO}(001)$
}

\author{
Aleksej Laptev, ${ }^{1}$ Kristof Lebecki, ${ }^{2}$ Gesa Welker, ${ }^{1, a)}$ Yuansu Luo, ${ }^{3}$ Konrad Samwer, ${ }^{3}$ \\ and Mikhail Fonin ${ }^{1, b)}$ \\ ${ }^{1}$ Fachbereich Physik, University of Konstanz, 78457 Konstanz, Germany \\ ${ }^{2}$ Nanotechnology Centre, VSB-TU Ostrava, 70833 Ostrava-Poruba, Czech Republic \\ ${ }^{3}$ I. Physikalisches Institut, Georg-August-Universität Göttingen, 37077 Göttingen, Germany
}

(Received 22 April 2016; accepted 11 September 2016; published online 28 September 2016)

\begin{abstract}
We study the temperature dependent magnetization properties of (010)-oriented Ni-Mn-Ga epitaxial films on $\mathrm{MgO}(001)$ substrates. In the martensitic phase, we observe pronounced abrupt slope changes in the magnetization loops for all studied samples. Our experimental findings are discussed in conjunction with the micromagnetic simulations, revealing that the characteristic magnetization behavior is governed solely by the magnetization switching within the specific martensitic variant pattern, and no reorientation of twin variants is involved in the process. Our study emphasizes the important role of the magnetostatic interactions in the magnetization behavior of magnetic shape memory alloy thin films. Published by AIP Publishing.

[http://dx.doi.org/10.1063/1.4963264]
\end{abstract}

Magnetic shape memory (MSM) alloys exhibit a remarkable functional property to generate macroscopic strains upon exposure to a magnetic field of sufficient strength. ${ }^{1,2}$ The strong coupling between the structural and magnetic degrees of freedom gives rise to the magnetically induced reorientation (MIR) of the twinned martensitic microstructure, resulting in a magnetic field-induced strain (MFIS). Strain values of up to $12 \%$ were reported for single crystals, ${ }^{3-5}$ boosting the research interest especially in MSM thin films, which have a strong application potential in sensing and actuation systems. ${ }^{6-8}$ In particular, the investigation of twin variant reorientation in epitaxial films has been the subject of several studies. However, the direct identification of the change of microstructure or the shape upon the application of external magnetic fields in thin films is a challenging task. Therefore, in analogy to bulk material studies, ${ }^{9}$ magnetization measurements have been utilized in order to identify the MIR of twin variants. The reorientation of martensitic variants is linked to the reorientation of the easy magnetization axis, resulting in a strong change of the measured magnetization of the sample.

A step-like increase in the magnetization has indeed been observed in constrained epitaxial thin films grown on different substrates. ${ }^{10-13}$ In analogy to bulk materials, this effect has also been identified as MIR of twin variants. However, a conclusive MIR model for thin films on a rigid substrate is still missing, owing to the fact that the substrate inhibits any macroscopic length change and thus hampers the magnetically induced change in the martensitic microstructure of an MSM film.

In this paper, we address the origin of steps in the magnetization loops of constrained epitaxial MSM films. We study the role of the martensitic microstructure and the magnetostatic interactions on the magnetization process of epitaxial Ni-Mn-Ga films on a rigid substrate. We employ

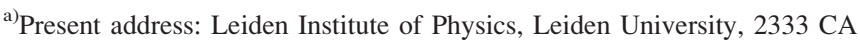
Leiden, The Netherlands.

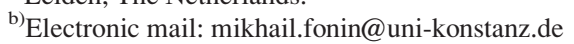

temperature-dependent magnetization measurements and micromagnetic simulations to develop a model, which consistently explains the peculiar magnetization behavior.

For this study, we use three epitaxial Ni-Mn-Ga film samples with different thicknesses (see Table I), prepared by DC magnetron sputtering. A co-sputtering method was applied using a target with the composition $\mathrm{Ni}_{48} \mathrm{Mn}_{31} \mathrm{Ga}_{21}$, and a Ni target. The Ni-Mn-Ga films were deposited at a substrate temperature of $773 \mathrm{~K}$ either directly onto the $\mathrm{MgO}(001)$ substrate (sample S1) or onto a $100 \mathrm{~nm}$ thick $\mathrm{Cr}(001)$ buffer layer grown at $823 \mathrm{~K}$ (samples S2 and S3). The films were grown in the parent austenitic phase with the epitaxial relations Ni-Mn-Ga(001)[100] $\| \mathrm{MgO}(001)[110]$ or Ni-Mn$\mathrm{Ga}(001)[100]\|\mathrm{Cr}(001)[100]\| \mathrm{MgO}(001)[110] .{ }^{14-17}$ The film compositions were determined by using energy dispersive $\mathrm{x}$ ray (EDX) spectroscopy. The magnetization curves as a function of the applied magnetic field along different orientations of the $\mathrm{MgO}$ substrate were measured by superconducting quantum interference device (SQUID) magnetometry at various temperatures. Magnetization as a function of temperature was measured to identify the Curie temperature. The martensitic transformation temperature $T_{\mathrm{M}}$ is supposed to be above $T_{\mathrm{C}}$ and was out of the temperature range of SQUID.

For micromagnetic simulations, we have used the OOMMF code. ${ }^{18}$ To avoid artifacts related to numerical space discretization, ${ }^{19}$ relatively small cells were used: $(2 \mathrm{~nm})^{3}$. Concerning the material parameters used in the simulation, we chose the experimental values obtained for the $7 \mathrm{M}$

TABLE I. Overview of the sample properties with $d$ being the film thickness, $T_{\mathrm{C}}$-the Curie temperature, $T_{\mathrm{M}}$-the martensitic transformation temperature, and $R_{\mathrm{Y}}$-the ratio of Y-type twinning.

\begin{tabular}{lccccc}
\hline \hline Name & $d(\mathrm{~nm})$ & Composition & $T_{\mathrm{C}}$ & $T_{\mathrm{M}}$ & $R_{\mathrm{Y}}$ \\
\hline S1 & 150 & $\mathrm{Ni}_{52} \mathrm{Mn}_{24} \mathrm{Ga}_{24}$ & $360 \mathrm{~K}$ & $>T_{\mathrm{C}}$ & $97 \%$ \\
S2 & 250 & $\mathrm{Ni}_{52} \mathrm{Mn}_{21} \mathrm{Ga}_{27}$ & $311 \mathrm{~K}$ & $>T_{\mathrm{C}}$ & $95 \%$ \\
S3 & 500 & $\mathrm{Ni}_{50} \mathrm{Mn}_{27} \mathrm{Ga}_{23}$ & $367 \mathrm{~K}$ & $>T_{\mathrm{C}}$ & $96 \%$ \\
\hline \hline
\end{tabular}


martensitic orthorhombic unit cell of bulk Ni-Mn-Ga samples. $^{20,21}$ Thus, anisotropy coefficients of $K_{\mathrm{a}}=-1.7 \times 10^{5}$ $\mathrm{J} \mathrm{m}^{-3}$ and $K_{\mathrm{b}}=-0.9 \times 10^{5} \mathrm{~J} \mathrm{~m}^{-3}$ were used for the magnetic hard $a$-axis and medium $b$-axis, respectively. A saturation magnetization of $M_{\mathrm{S}}=4.88 \times 10^{5} \mathrm{~A} \mathrm{~m}^{-1}$ and an exchange constant of $A=10^{-12} \mathrm{~J} \mathrm{~m}^{-1}$ were used. Simulating the hysteresis, the cells were initially randomly magnetized; the field was applied with a small tilt of $0.7^{\circ}$ to the desired direction and the system was allowed to relax. These steps were performed to avoid unintentional symmetries. ${ }^{22}$ Due to the high computational time, only one half of the hysteresis cycle was calculated.

The magnetization curves of sample S3 obtained at room temperature in the martensitic state are presented in Fig. 1(a). The curves were measured normal $(H|| \mathrm{MgO}[001])$ and parallel $(H|| \mathrm{MgO}[100]$ and $H|| \mathrm{MgO}[110])$ to the film plane. The magnetization loop measured along the $\mathrm{MgO}[100]$ direction demonstrates abrupt changes in the slope for magnetic fields near the field values $H_{\mathrm{S} 1}, H_{\mathrm{C}}$, and $H_{\mathrm{S} 2}$. The step-like shape of the hysteresis can be well recognized in the enlarged view shown in Fig. 1(b). A similar behavior of the magnetization was observed for all studied samples for temperatures below $T_{\mathrm{C}}$ as can be observed in Figs. 1(b) and 1(c). The measurement temperatures were chosen in the way to have a comparable separation from $T_{\mathrm{C}}$ for each sample. Fig. 1(c) demonstrates that the overall shape of the magnetization curve is not altered upon going to low temperatures. However, the effect occurs at higher values of the applied magnetic field. Fig. 1(d) shows the temperature dependence of the switching field. The average values of $H_{\mathrm{S} 1}$ and $H_{\mathrm{S} 2}$, which are defined as the position of the largest slope change in magnetization, are displayed.
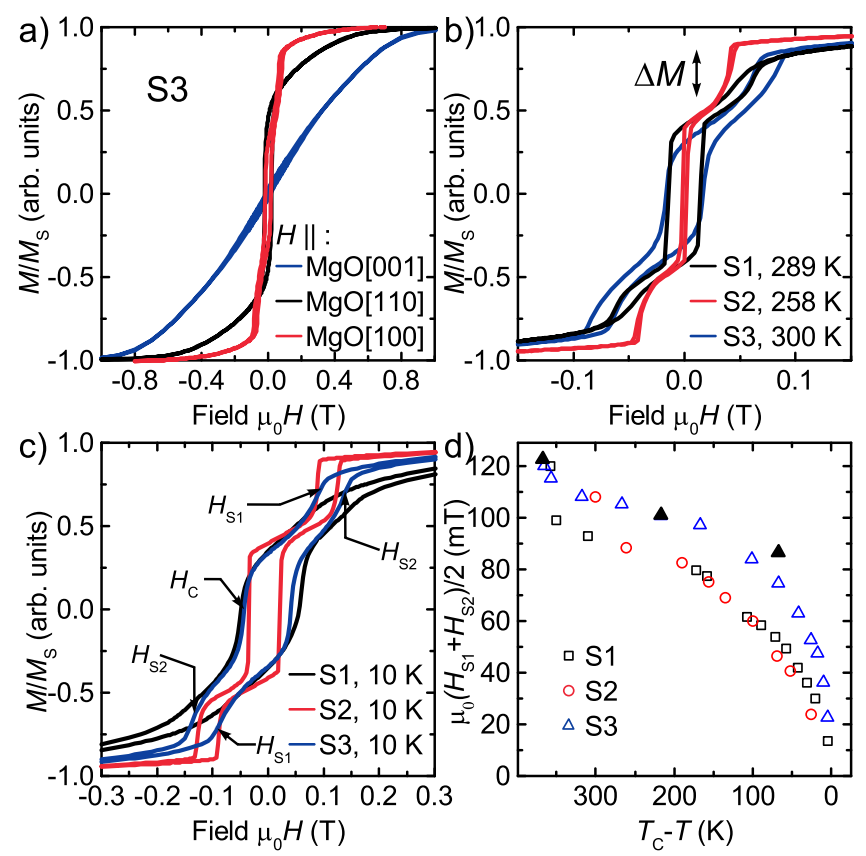

FIG. 1. (a) Hysteresis curves obtained in the martensitic state at $300 \mathrm{~K}$ for sample S3. The blue curve was measured perpendicular to the film plane and was not corrected for the demagnetization field. (b) and (c) Magnetization curves measured for samples $\mathrm{S} 1, \mathrm{~S} 2$, and S3 along the $\mathrm{MgO}[100]$ direction at $289 \mathrm{~K}(\mathrm{~S} 1), 258 \mathrm{~K}(\mathrm{~S} 2)$, and $300 \mathrm{~K}$ (S3) and at $10 \mathrm{~K}$ (all samples). (d) Measured temperature dependence of the switching field displayed as an anhysteretic value: $\left(H_{S 1}+H_{S 2}\right) / 2$ (open symbols). Filled symbols represent the calculated values.
For all samples, the effect occurs at similar switching fields and shows a similar temperature behavior. As the temperature is decreased, the switching field increases.

The described shape of the magnetization curve, the values of the switching field, and also its temperature behavior have been observed in a number of constrained epitaxial thin films and attributed to the MIR effect. ${ }^{10-13}$ It is remarkable that the reported values of the switching field are similar despite different growth techniques, sample orientations, and used substrates. In the following, we will show that this behavior can be explained on the basis of the model, which assumes the martensitic twin variant structure as fixed and only considers the magnetostatic interactions and the evolution of magnetic domains.

Epitaxial martensitic Ni-Mn-Ga films show a characteristic microstructural pattern at the film surface defined by the twin boundaries (TB), which cross the film surface. ${ }^{14,16,17}$ These TB are clearly visible as linear traces in scanning electron microscopy (SEM) images as shown in Fig. 2(a). Depending on the orientation of the $\{101\}$-TB with respect to the film surface $\left(45^{\circ}\right.$ or $\left.90^{\circ}\right)$ and the direction of the film surface intersection (along $\mathrm{MgO}[110]$ or $\mathrm{MgO}[100]$, respectively), two types of twinning configuration can be identified. Using the nomenclature introduced in Ref. 17, the two different configurations will be labeled as $\mathrm{Y}$-type $\left(90^{\circ}\right)$ and $\mathrm{X}$-type $\left(45^{\circ}\right)$. Both twinning types are observed simultaneously, as can be seen in Fig. 2(a); however, Y-type twinning dominates in all studied samples (see Table I). The possible TB
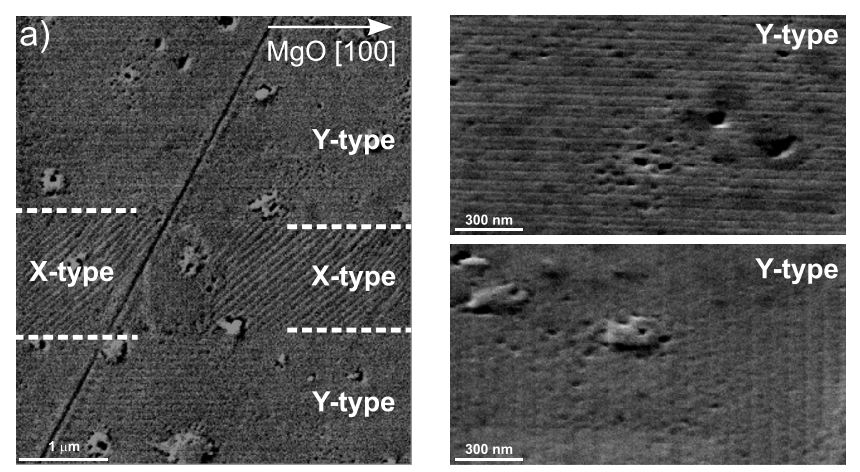

b) Y-type

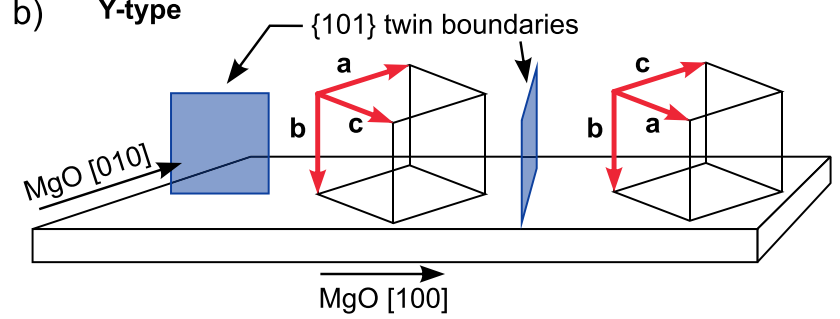

c)

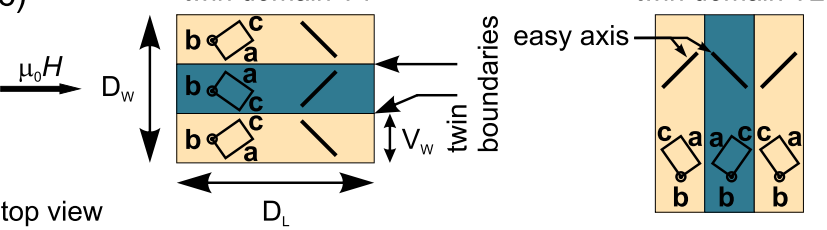

FIG. 2. (a) Martensitic microstructure of sample S3 as visible in the SEM. Mainly the Y-type twinning configuration is present in the sample. (b) Sketch of the unit cell and twin boundary orientation within the Y-type twinning configuration. (c) Schematic drawing of the twinning pattern arrangement for the Y-type twinning. 
orientations within the Y-type twinning are shown in Fig. 2(b). We note a specific orientation of the short $c$-axis, which is the easy magnetization axis of the martensitic unit cell. The $c$-axis lies always within the film plane and points along the $\mathrm{MgO}\langle 110\rangle$ direction. At twinning planes, the $c$ - and $a$ axes change their direction by $90^{\circ}$, while the $b$-axis is always oriented perpendicular to the film plane.

In order to study the influence of this twinning pattern arrangement on the magnetization behavior, micromagnetic simulations have been performed. In the simulation, the sample with the thickness of $126 \mathrm{~nm}$ is comprised of two twin domains (Y1 and Y2) [see Fig. 2(c)]. Each twin domain had a width of $D_{\mathrm{W}}=968 \mathrm{~nm}$ and a length of $D_{\mathrm{L}}=2 \mu \mathrm{m}$. The domains are comprised of twin variants with the width of $V_{\mathrm{W}}=88 \mathrm{~nm}$. For comparison, the experimental values for sample S3 obtained from SEM measurements are: $D_{\mathrm{W} \mid \mathrm{S} 3} \approx 2 \mu \mathrm{m}, D_{\mathrm{L} \mid \mathrm{S} 3} \approx 6 \mu \mathrm{m}$, and $V_{\mathrm{W} \mid \mathrm{S} 3} \approx 89 \mathrm{~nm}$. The direction of the external magnetic in-plane field was pointing along the TB for the twin domain Y1 and perpendicular to the TB for the twin domain Y2. This orientation of the magnetic field corresponds to the $\mathrm{MgO}$ [100] direction in the experiment. The microstructure is fixed in the simulation, i.e., no TB movement is allowed.

Fig. 3 shows the simulated magnetic domain structure and the magnetization curve. The latter reproduces the experimentally obtained hysteresis curve very well. The coinciding saturation behavior suggests that the material parameters used in the simulation are in good agreement with sample properties. Of more importance is the fact that the simulated curve exhibits the step-like shape with three distinct magnetization steps. The reason for the appearance of the three steps is revealed by the micromagnetic simulations, as presented in Figs. 3(b)-3(e). The first step around $H_{\mathrm{S} 1}$ occurs between state (b) and (c). At a magnetic field of $120 \mathrm{mT}$, twin domain Y2 exhibits a staircase domain pattern, where the magnetization is confined to the $c$-axis in each variant (Fig. 3(b)). On the contrary, for twin domain Y1 the situation is more complex. For this twin variant configuration, where the TB are aligned parallel to the direction of the magnetic field, the magnetization rotation towards the easy magnetization axis results in a configuration with head-to-head and tail-to-tail domain walls at the TB. As the applied magnetic field is decreased, the first step in the magnetization curve occurs due to magnetization orientation change within the twin domain Y1. As can be observed in Figs. 3(b) and 3(c), the magnetization switches for every second twin variant. This switching is clearly related to a nucleation of $180^{\circ}$ domain walls at the twin domain boundary. As we are not able to mimic the exact experimental conditions (edge roughness/structure) with our simulations and as we do not take into account the inter-twin-domain interactions, the exact value of this switching field does not precisely meet our experimental result. The resulting magnetic domain configuration at nearly zero field is depicted in Fig. 3(c). The magnetization follows the easy axis in each martensitic variant and the overall pattern resembles the magnetic staircase pattern. We would like to emphasize that this domain pattern type exactly reproduces the magnetic structure, which has
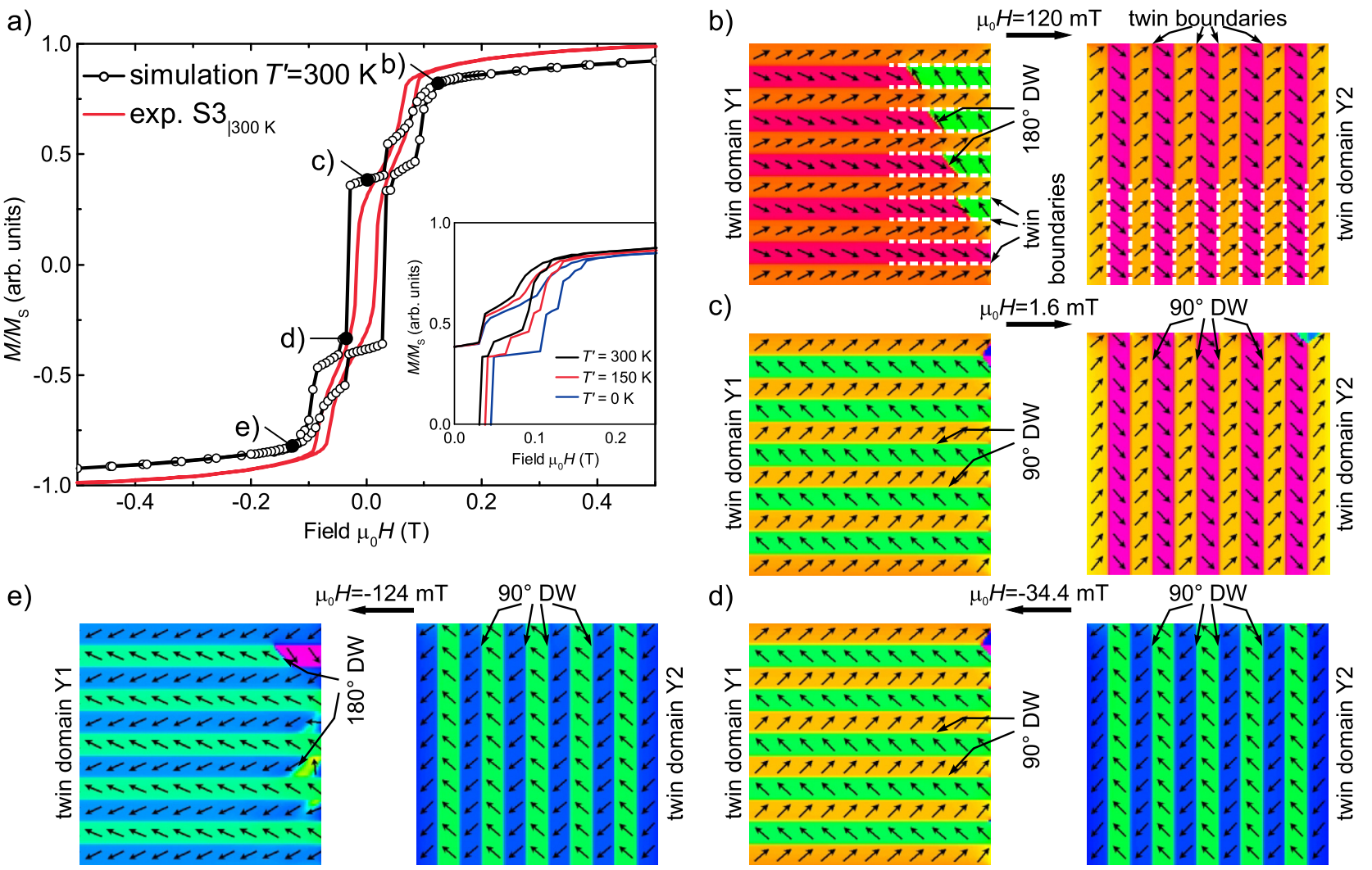

FIG. 3. (a) Magnetization curve obtained from the micromagnetic simulations. The experimental hysteresis curve obtained for sample $\mathrm{S} 3$ is shown in red for comparison. The inset shows the first quadrant of the magnetization curves calculated for different temperatures. $T^{\prime}$ denotes a simulated temperature. (b)-(e) Simulated magnetic domain microstructure evolution within both twin domains Y1 and Y2 during the magnetization process for specific magnetic fields. 
previously been observed both experimentally ${ }^{13,17,23}$ at zero field in Ni-Mn-Ga films with similar martensitic structure and by comparable micromagnetic simulations. ${ }^{23}$

The second step at $H_{\mathrm{C}}$ is related to a magnetization configuration change solely in twin domain Y2 (states (c) and (d)). This step is the most distinctive one, because the magnetization switches for every twin variant of this twin domain type. The third step around $H_{\mathrm{S} 2}$ occurs due to the switching in twin domain Y1 (state (d) and (e)). Again, the magnetization switches only for every second twin variant in order to align the magnetization configuration of Y1 along the magnetic field.

The peculiar hysteresis shape of the sample emerges due to the specific orientation of the easy axis and the TB, which define the position of the $90^{\circ}$ magnetic domain walls, with respect to the magnetic field direction. The head-tohead magnetization pattern can form during the magnetization process only if TB are aligned along the magnetic field and the magnetic easy axis is oriented at $45^{\circ}$ to the magnetic field. This situation occurs only if the magnetic field is applied along the $\mathrm{MgO}[100]$ direction. Therefore, no similar step-like behavior in the hysteresis loops can be detected for other field directions, i.e., for $H \| \mathrm{MgO}[110]$ and $H \| \mathrm{MgO}[001]$ (see Fig. 1(a)).

In their work on steps in the magnetization loops of epitaxial Ni-Mn-Ga films, Thomas et al. presented an analytical model, which describes the possibility of the MIR in films with constrained overall extension. ${ }^{10}$ This model is based on the fact that an orthorhombic structure allows three free parameters for the orientation of the unit cell. Hence, in an applied magnetic field, a reorientation of the unit cells is possible if the fraction of the $a$-variants (longest lattice constant) is reduced when the fraction of $b$-variants (medium lattice constant) increases and the fraction of $c$-variants (shortest lattice constant) is reduced at the same time. This process requires no overall length change of the constrained film. However, several questions remain open. First, the observed magnetization loop steps appear at magnetic fields, which are around one order of magnitude lower (Fig. 1(d)) compared to the field values at which the MIR occurs in best single crystals. ${ }^{3-5}$ Second, the observed temperature behavior of the switching field in the epitaxial films requires some explanation. It has been suggested that the switching values decrease with the temperature due to thermally activated reduction of the twinning stress. ${ }^{11}$ However, experiments show that twinning stress strongly increases with the decreasing temperature. On the contrary, magnetization loop steps can be observed at temperatures down to $10 \mathrm{~K}$ (Fig. $1(\mathrm{c}))$. In the following, we will show that the temperature behavior of the switching field can be explained by the temperature behavior of the material parameters.

In order to calculate the hysteresis curves for different temperatures, the temperature dependence of the material parameters used in the simulations was mimicked. The material parameters quoted at the beginning were set as room temperature parameters $M_{\mathrm{S} \mid 300 \mathrm{~K}}^{\prime}, A_{\mid 300 \mathrm{~K}}^{\prime}$, and $K_{\mathrm{a}, b \mid 300 \mathrm{~K}}^{\prime}$. The saturation magnetization was scaled according to the experimentally obtained magnetization temperature behavior of sample $\mathrm{S} 3: M_{\mathrm{S} \mid 150 \mathrm{~K}}^{\prime}=5.4 \times 10^{5} \mathrm{Am}^{-1}, M_{\mathrm{S} \mid 0 \mathrm{~K}}^{\prime}=6.1$ $\times 10^{5} \mathrm{Am}^{-1}$. The values for the exchange constant and the anisotropy coefficients were scaled according to published data: ${ }^{20,24} \quad A_{\mid 150 \mathrm{~K}}^{\prime}=1.16 \times 10^{-12} \mathrm{Jm}^{-1}, A_{\mid 0 \mathrm{~K}}^{\prime}=1.4 \times 10^{-12} \mathrm{Jm}^{-1}$, $K_{\mathrm{a} \mid 150 \mathrm{~K}}^{\prime}=-2.2 \times 10^{5} \mathrm{~J} \mathrm{~m}^{-3}, K_{\mathrm{a} \mid 0 \mathrm{~K}}^{\prime}=-3.0 \times 10^{5} \mathrm{~J} \mathrm{~m}^{-3}$ and $K_{\mathrm{b} \mid 150 \mathrm{~K}}^{\prime}=-1.16 \times 10^{5} \mathrm{~J} \mathrm{~m}^{-3}, K_{\mathrm{b} \mid 0 \mathrm{~K}}^{\prime}=-1.6 \times 10^{5} \mathrm{~J} \mathrm{~m}^{-3}$. The results of the simulations are presented in the inset of Fig. 3(a), where the first quadrant of the simulated magnetization curves is shown. The micromagnetic simulations excellently reproduce the temperature behavior observed in the experiment. As the temperature is decreased, the theoretically obtained switching fields $H_{S 1}$ and $H_{S 2}$ steadily increase. This qualitatively reproduces the temperature behavior experimentally observed in this study (see Fig. 1(d)) and in published data. ${ }^{11,12}$

In conclusion, we have examined the temperature dependent magnetization properties of Ni-Mn-Ga epitaxial films in the martensitic phase. Micromagnetic simulations have demonstrated that the abrupt changes observed in the hysteresis slope originate from the magnetization switching within the martensitic variant pattern. The occurrence of such specific hysteresis loops is facilitated by the existing twinned microstructure, which features a periodic orientation change of the magnetic easy axis on the nanometer scale. The results presented here reveal the fact that the step-like magnetization behavior of Ni-Mn-Ga epitaxial films does not necessarily point to a magnetically induced reorientation of the martensitic variants. The complex magnetostatic interactions within the martensitic microstructure have to be taken into account in order to correctly interpret the peculiar magnetization behavior of magnetic shape memory alloy materials.

${ }^{1}$ K. Ullakko, J. K. Huang, C. Kantner, R. C. O'Handley, and V. V. Kokorin, Appl. Phys. Lett. 69, 1966 (1996).

${ }^{2}$ Q. Pan and R. D. James, J. Appl. Phys. 87, 4702 (2000).

${ }^{3}$ A. Sozinov, A. A. Likhachev, N. Lanska, and K. Ullakko, Appl. Phys. Lett. 80, 1746 (2002).

${ }^{4}$ A. Sozinov, N. Lanska, A. Soroka, and W. Zou, Appl. Phys. Lett. 102, 021902 (2013).

${ }^{5}$ E. Pagounis, M. J. Szczerba, R. Chulist, and M. Laufenberg, Appl. Phys. Lett. 107, 152407 (2015).

${ }^{6}$ M. Thomas, O. Heczko, J. Buschbeck, Y. W. Lai, J. McCord, S. Kaufmann, L. Schultz, and S. Fähler, Adv. Mater. 21, 3708 (2009).

${ }^{7}$ M. Schmitt, A. Backen, S. Fähler, and M. Kohl, Microelectron. Eng. 98, 536 (2012).

${ }^{8}$ M. Kohl, M. Schmitt, A. Backen, L. Schultz, B. Krevet, and S. Fähler, Appl. Phys. Lett. 104, 043111 (2014).

${ }^{9}$ O. Heczko, J. Magn. Magn. Mater. 290-291, 787 (2005).

${ }^{10}$ M. Thomas, O. Heczko, J. Buschbeck, U. K. Rößler, J. McCord, N. Scheerbaum, L. Schultz, and S. Fähler, New J. Phys. 10, 023040 (2008).

${ }^{11}$ Y. Zhang, R. A. Hughes, J. F. Britten, J. S. Preston, G. A. Botton, and M. Niewczas, Phys. Rev. B 81, 054406 (2010).

${ }^{12}$ R. Xie, S.-L. Tang, Y.-M. Tang, X.-C. Liu, T. Tang, and Y.-W. Du, Chin. Phys. B 22, 107502 (2013).

${ }^{13}$ P. Ranzieri, M. Campanini, S. Fabbrici, L. Nasi, F. Casoli, R. Cabassi, E. Buffagni, V. Grillo, C. Magen, F. Celegato, G. Barrera, P. Tiberto, and F. Albertini, Adv. Mater. 27, 4760 (2015).

${ }^{14}$ P. Leicht, A. Laptev, M. Fonin, Y. Luo, and K. Samwer, New J. Phys. 13, 033021 (2011).

${ }^{15}$ Y. Luo, P. Leicht, A. Laptev, M. Fonin, U. Rüdiger, M. Laufenberg, and K. Samwer, New J. Phys. 13, 013042 (2011).

${ }^{16}$ T. Eichhorn, R. Hausmanns, and G. Jakob, Acta Mater. 59, 5067 (2011).

${ }^{17}$ A. Diestel, V. Neu, A. Backen, L. Schultz, and S. Fähler, J. Phys. Condens. Matter 25, 266002 (2013). 
${ }^{18}$ M. J. Donahue and D. G. Porter, OOMMF User's Guide, Version 1.0: Interagency Report (National Institute of Standards and Technology, Gaithersburg, MD, 1999).

${ }^{19}$ K. M. Lebecki and U. Nowak, Phys. Rev. B 89, 014421 (2014).

${ }^{20}$ L. Straka and O. Heczko, J. Appl. Phys. 93, 8636 (2003).

${ }^{21}$ V. Runov and U. Stuhr, J. Magn. Magn. Mater. 323, 244 (2011).
${ }^{22}$ K. M. Lebecki, O. Kazakova, and M. W. Gutowski, Physica B 403, 360 (2008).

${ }^{23}$ A. M. Jakob, M. Hennes, M. Müller, D. Spemann, and S. G. Mayr, Adv. Funct. Mater. 23, 4694 (2013).

${ }^{24}$ N. Kazantseva, D. Hinzke, U. Nowak, R. W. Chantrell, U. Atxitia, and O. Chubykalo-Fesenko, Phys. Rev. B 77, 184428 (2008). 